\title{
Parvo Viruses Complexed With Neutralizing Antibody Fragments Studied by Cryo Electron Microscopy and Three-Dimensional Image Reconstruction
}

\author{
V.D. Bowman, ${ }^{*}$ S. Hafenstein, ${ }^{*}$ J. Nelson, $^{*}$ P.R. Chipman, ${ }^{*}$ A.J. Battisti, ${ }^{*}$ L. Palermo, ${ }^{* *}$ C. Nelson, ${ }^{* *}$ \\ C. Parrish, ${ }^{* *}$ and M.G. Rossmann \\ *Department of Biological Sciences, Purdue University, West Lafayette, IN 47907-1392 \\ ** J.A. Baker Institute, Department of Microbiology and Immunology, College of Veterinary \\ Medicine, Cornell University, Ithaca, NY 14853
}

The immune response launched by animals resulting from virus infection is complicated and multifaceted. Neutralization of viruses by host antibodies may utilize multiple approaches both independently and simultaneously; including aggregation of virus particles, interfering with binding to cellular receptors and endocytosis, inducing changes in the capsid structure, or halting viral genome replication by prohibiting uncoating. In our efforts to gain insight into the process of antibody binding and neutralization, we used electron cryo-microscopy (cryo EM) and threedimensional (3D) image reconstruction methods to study the interaction of two Parvoviruses, feline panleukopenia virus (FPV) and canine parvovirus (CPV) complexed with neutralizing monoclonal antibody fragments (Fabs) [1].

These small (260 angstroms in diameter) viruses make an excellent model system to study antibody/virus interactions as they are non-enveloped and icosahedral; making them particularly amendable to cryo EM and to 3D reconstruction methods utilizing symmetry. Atomic structures of FPV [2] and CPV [3] are available, and well characterized biochemically. The capsid is assembled from 60 copies of 1 structural motif, which is found in the overlapping structural proteins VP1, VP2, and VP3. Each of the 60 subunits contains the eight-stranded antiparallel beta-barrel motifs found in many other known viral capsid structures $[4,5]$ but with large insertions between some beta-strands. These loops form much of the capsid surface, including the threefold spikes (Fig.1.B, black arrows). The spike region contains residues involved in host recognition and antigenicity [6].

Mutations in the parvo viral capsid protein can result in mutants that maintain infectivity in the presence of neutralizing antibodies [6]. Escape mutants of FPV against a series of monoclonal antibodies raised to FPV identified individual amino acid residues likely to be involved in binding to specific IgGs. The escape mutants clustered into 2 areas on the capsid surface, termed sites A and B. Site A is near the three-fold axis of symmetry while the B site is closer to the two-fold axis [1]. Eight monoclonal antibodies were chosen for further study and used to generate the Fabs used in this study. Cryo-EM and image reconstructions confirmed the postulated major (A and B) binding sites of these antibodies, one near the three-fold axis of symmetry (Fig.1.A, black arrowhead) corresponding to site A, and one closer to the two-fold axis (Fig.1.B, black arrowheads) in the region of site B.

Samples of purified virus were incubated with an excess of Fabs to ensure saturation and then prepared for cryo electron microscopy as described [7]. Images were recorded with under low dose conditions in a Philips CM300 FEG transmission electron microscope at a nominal magnification of 47,000x. Micrographs [Table 1] were recorded over a range of defocus levels (1-3microns defocus), digitized and bin averaged to a pixel size of 3.1 langstroms and analyzed by three-dimensional 
reconstruction methods [7]. Reconstructions were computed from selected particle images and ranged from 8-16 angstroms resolution (Fig.1). The effects of the microscope contrast transfer function were partially corrected in the Fourier transform of each image.

\section{References:}

[1] W. R. Wikoff et al., Struct. 2 (1994) 595.

[2] M. Agbanje et al., Proteins, 16 (1993) 155

[3] Q. Xie and M. S. Chapman, J. Mol. Biol., 264 (1996) 497.

[4] M. G. Rossman and J. E. Johnson, Annu. Rev. Biochem., 58 (1989) 533.

[5] N. Nandhagopal et al., Proc. Natl. Acad. Sci. U.S.A. 23 (2002) 14758.

[6] M. L. Strassheim et al. Virol. 198 (1994) 175.

[7] T. S. Baker et al., Microbiol. Molec. Biol. Reviews, 63 (1999) 862.

[8] Work supported in part by grants from the NIH to CP (AI33468), SH (A160155), to MGR (AI11219) a Program Project Grant to Richard Kuhn (AI55672); a Fulbright scholarship to JN; and awards to the Purdue Structural Biology Group from the Keck Foundation and Purdue University.
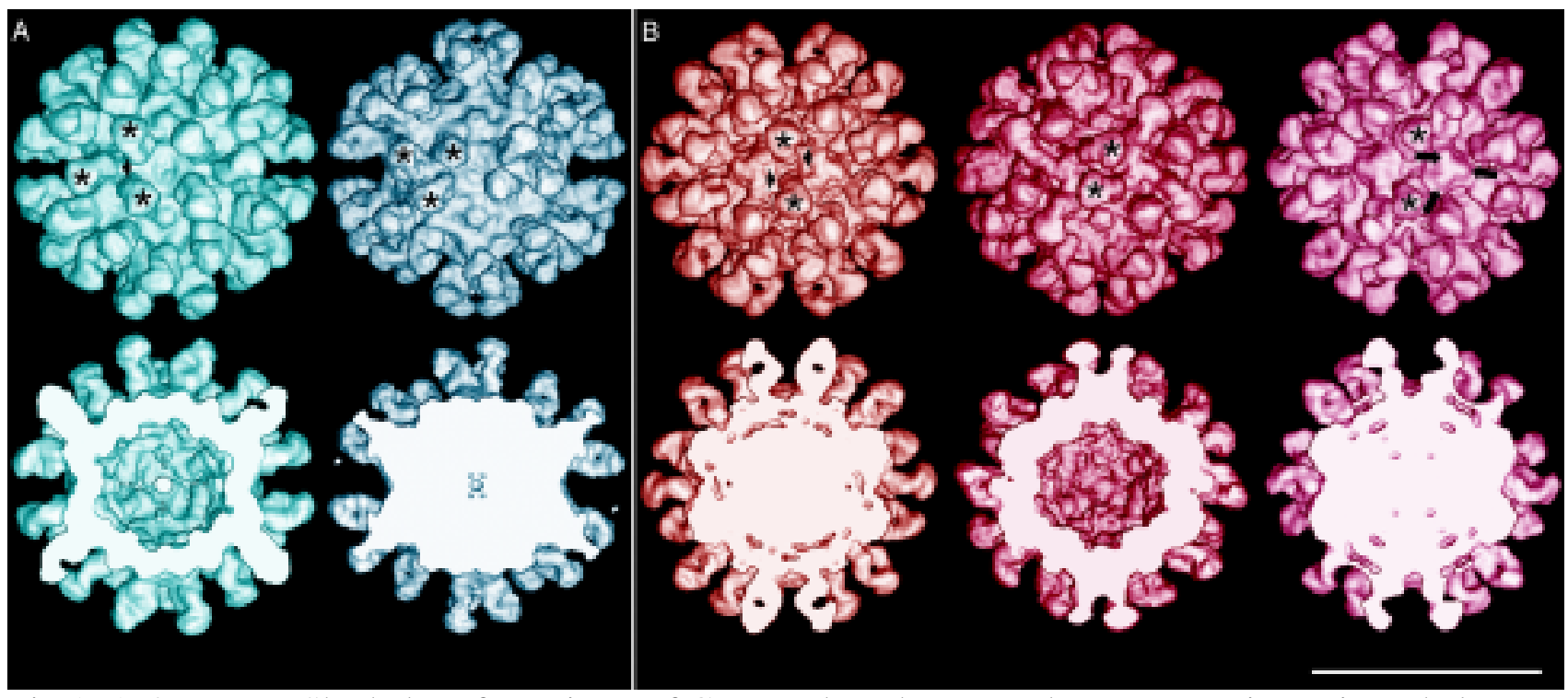

Fig.1. A \& B Top, Shaded-surface views of CPV+Fab and FPV+Fab reconstructions viewed along twofold axes. A \& B Bottom, same views with the front half removed. A. Complexes of virus with A site binding Fabs. Cyan, CPV+Mab A4E3, Steel blue, FPV+Mab B2E3 B. Complexes of virus with B site binding Fabs. Red, FPV+Mab A3B10, Magenta, FPV+Mab B6D5, Fuschia, FPV+Mab A4E4. Asterisks mark the distal end of bound Fabs. All reconstructions were calculated to 15 angstroms for comparison. Scale bar: 250 angstroms.

TABLE 1. Particle processing data
\begin{tabular}{|lccccc|}
\hline $\begin{array}{l}\text { Complex } \\
\text { A site }\end{array}$ & Mab Designation & Micrographs & Boxed Particles & Map Particle Number & Resolution in Angstroms \\
FPV+MabB & B2E3 & 56 & 1576 & 1126 & 15 \\
CPV+Mab14 & A4E3 & 139 & 2800 & 2700 & 15 \\
B site & & & & & 8.8 \\
FPV+Mab8 & A3B10 & 49 & 6480 & 4344 & 12 \\
FPV+MabF & B6D5 & 93 & $\mathbf{2 4 5 0}$ & $\mathbf{1 6 9 0}$ & 10.5 \\
FPV+Mab15 & A4E4 & $\mathbf{5 0}$ & $\mathbf{6 9 5 5}$ & $\mathbf{4 7 9 8}$ & \\
\hline
\end{tabular}

